JOURNAL OF

SYNCHROTRON

RADIATION

ISSN 1600-5775

\section{Synchrotron Radiation and Free-Electron Lasers. Principles of Coherent X-ray Generation. By Kwang-Je Kim, Artur Braun and Zhirong Huang. Cambridge University Press, 2017. Pp. 298. Price GBP 89.99, hardback, ISBN 978-1107162617.}

\author{
John R. Helliwell*
}

School of Chemistry, The University of Manchester, Brunswick Street, Manchester M13 9PL, UK.

${ }^{*}$ Correspondence e-mail: john.helliwell@manchester.ac.uk

Keywords: book review;

synchrotron radiation; FEL radiation.

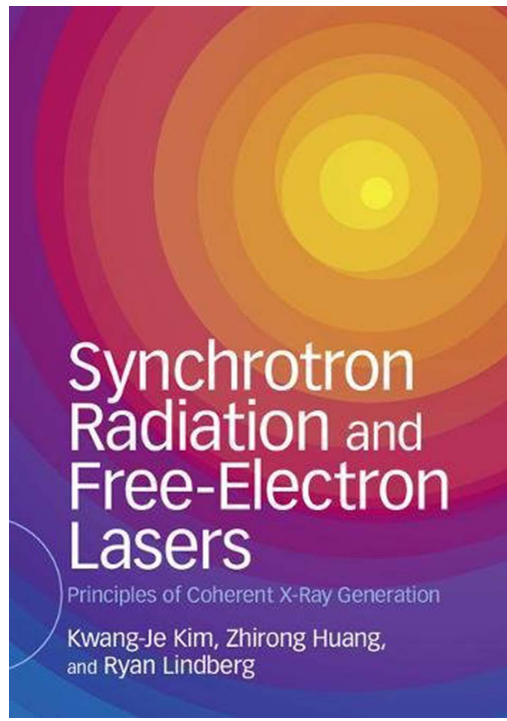

(C) 2018 International Union of Crystallography
When I represented the European Synchrotron Radiation Facility (ESRF) Science Advisory Committee at the ESRF Machine Advisory Committee meetings in the early 1990s I had the privilege to meet many of the leading accelerator physicists of the time. ESRF was the first third-generation synchrotron radiation (SR) source in the world and experience of such X-ray light sources and the emission properties in practice was naturally limited. Machine physicists at the meetings took an approach which to me was an amalgam of scientist and of engineer; the latter tempering science theory and experiments with what I call safe parameter specifications of the engineer ('safety only of the fundamental and third harmonic, and also assuming a minimum undulator magnet gap of $20 \mathrm{~mm}$. I commented that "couldn't the higher harmonics be added?" and the reply was that they could not be sure of the precision of the periodicity of the placement of each successive magnet, to which I replied 'it seems like the perfection of a crystal and trying to achieve higher diffraction orders'. Their reply was that shimming of magnets to extend to higher harmonics might be possible. With respect to the undulator minimum gap of $20 \mathrm{~mm}$, smaller undulator gaps were to prove possible, i.e. while still retaining safe electron injection.

It is with such a background that I really looked forward to reviewing this book, and which I have enjoyed. I learned in detail from the book that the discipline of SR machine physics experience has come a long way, and has been firmly extended into the domain of X-ray viable free-electron lasers (FELs). The back cover of the book promises 'a comprehensive resource for graduate students, researchers and practitioners who design, manage or use X-ray facilities'.

This book, to say the least, is for the mathematically trained. It certainly brought back memories of the mathematical physics courses of my undergraduate years at York University as well as, of course, resonating with many aspects of my work as a SR facility user and provider, through the different stages of my scientific career.

The authors' view of what is the most interesting aspect of SR in their book was quite revealing; for example, on page 33, 'modern synchrotron facilities, often referred to as light sources, use high-quality electron beams to provide a high-brightness flux of photons in the soft to hard X-ray region of the spectrum that is not accessible with other sources'. They do not actually say 'fully tunable' or 'polychromatic'. Nor can one find these words in the contents list or subject index. Undulator radiation and their emission lines are a key aspect, I agree, but these emission lines are moved around by varying the magnet gap so as to achieve tunability. Fig. 2.1 does explicitly show the polychromatic nature of the bending magnet and the wiggler though. The figure could be improved by showing numbers on the $y$-axis, i.e. to emphasize the higher intensity of the wiggler. Also the wavelength shifter as a type of SR source could have been illustrated. The strength of this book is, as I remarked earlier, the extensive mathematical treatments, but in turn the accessibility of the book text to SR and FEL facility users relies on figures like Fig. 2.1.

The authors' book subtitle stresses the principles of coherent X-ray generation and this is indeed brought out in various aspects such as longitudinal and transverse coherence factors'). Thus, I saw undulator X-ray emission spectra tabled for discussion consisting 
including coherence length and time. Their illustration with respect to Young's two-slit interference experiment is a neat didactic touch.

Errors in engineering of magnets are treated well. The text leaps into life at page 102 with its concluding section stating: 'we would like to emphasize that X-ray FELs based on SASE (self-amplified spontaneous emission) would not have been realised without incredible improvements in the production, transport and manipulation of electron beams, since very high brightness electron beams are essential for X-ray FELs ... made possible by photocathode gun design ... and tremendous improvements of (radiofrequency) RF linac and undulator technology'. Not only did I see for myself on the ESRF Machine Advisory Committee the early days of understanding $\mathrm{X}$-ray undulators, as I mentioned at the start of this review, but I had also seen the early days of installing the superconducting (5 T) wavelength shifter (SCW) in straight nine of the UK SRS. When I reported to the SRS Machine Control Room that when the SCW was ramped up to $5 \mathrm{~T}$ my X-ray beam intensity at the sample on SRS PX 7.2 halved, I was told that could not be so. Thus, by measuring the X-ray beam as far away from the tangent point of bending magnet 7 using green paper placed in the experimental hutch of SRS 7.4 (at the time not assigned), I could show directly the doubled size exposed vertical beam, due obviously to the doubling of the X-ray beam divergence. About a month later it was announced that the SRS SCW 9 had been placed slightly off the horizontal plane (by $0.75^{\circ}$ ) and the tilt would be rectified. Sure enough when this was done the SRS SCW 9 no longer affected my beam intensity on SRS PX7.2! For the modern day, pages 216-220 of this book very nicely capture the topics of 'undulator errors and tolerances' and 'beam trajectory errors'. I would like to imagine that equally astute users contributed to the knowledge and understanding of those topics as I did to our SRS SCW 9.

The book's allocation of pages is by far predominantly disposed to FELs. More could have been allocated to synchrotron radiation I think, for example to explain the wave of upgrades ongoing in the third-generation SR sources. Yes, in principle, the multibend achromat lattice (\$2.5.1) is covered, but the practical intricacies of, for example, the Extremely Brilliant Source project of the ESRF would be very interesting and could be included I think in a second edition.

To summarize, this book is a feast of mathematical physics and deserves extensive study to fully understand synchrotron radiation and FELs, and their coherence emission, in all their glories. Their development is one of the most remarkable scientific and technological advances of all of the last 50 years or so. 